\title{
Extracorporeal membrane oxygenator as a bridge to heart-liver en bloc transplant in a patient with Fontan circulation
}

\author{
Jennifer Lynn Kim, MD, ${ }^{a}$ Sumeet S. Vaikunth, MD, ${ }^{\mathrm{b}}$ Christiane Haeffele, MD, ${ }^{\mathrm{b}}$ and \\ John W. MacArthur, MD, ${ }^{\text {a }}$ Stanford, Calif
}

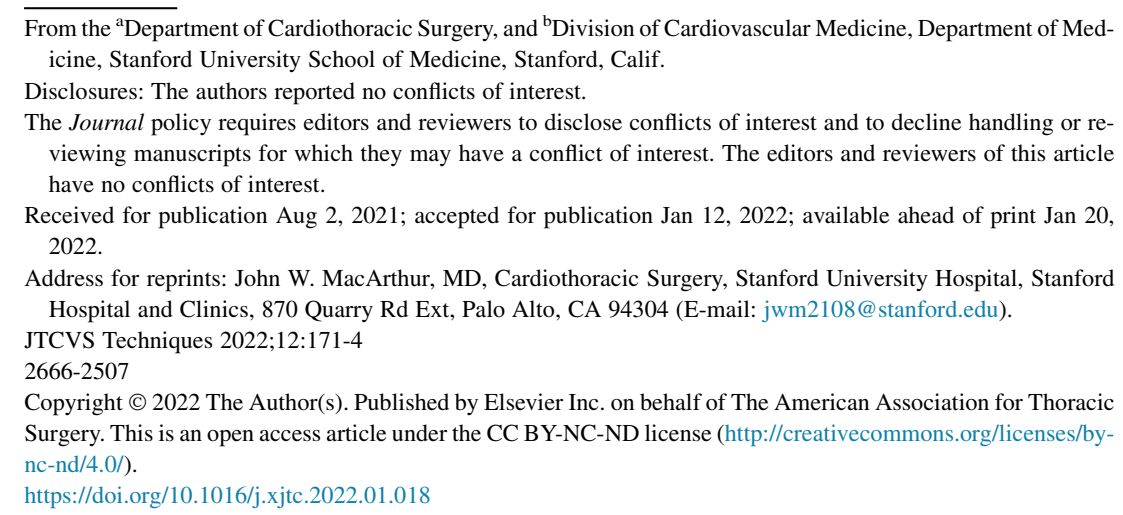

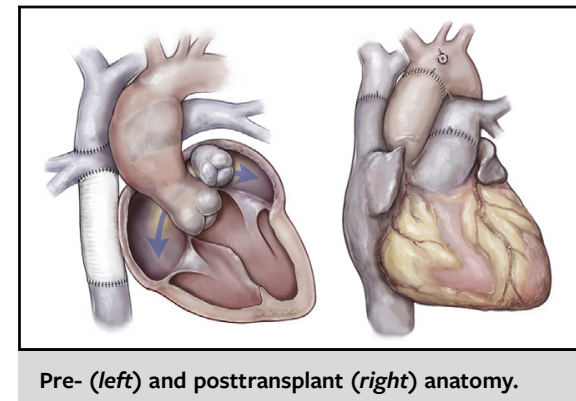

CENTRAL MESSAGE

Venoarterial extracorporeal

membrane oxygenation can be used as a bridge to heart-liver en bloc transplant in patients with Fontan circulation.

See Commentary on page 175 circulation. Here, we report the first use of ECMO as a bridge to heart-liver en bloc transplant in a female with a history of right ventricle-dominant atrioventricular septal defect who underwent a Fontan procedure (patient consent obtained, institutional review board \#295-22; January 3, 2019).

\section{CASE REPORT}

The patient is a 44-year-old female with a history of right ventricle-dominant atrioventricular septal defect who underwent pulmonary artery banding as an infant followed by a combined bidirectional Glenn procedure and extracardiac Fontan at age 22 years. Over the subsequent years, she developed arrhythmias, ascites, and symptoms of right heart failure with hemodynamics consistent with Fontan failure with normal pressures (Table E1). ${ }^{2}$ She was hospitalized for arrhythmias and started on milrinone. At this time, she was found to have radiographic evidence of cirrhosis, biopsy showing steatosis with pericentral fibrosis and focal early bridging, and severe ascites; therefore, she was listed for heart-liver transplant.

During her hospitalization, she had recurrent ascites that required frequent paracenteses. She developed spontaneous bacterial peritonitis and was treated with broadspectrum antibiotics. Due to persistent arrhythmias and
Although there is excellent long-term survival of patients
who have had a Fontan procedure for single-ventricle physiology, many of these patients eventually develop Fontan failure and require heart and, possibly, liver transplantation. $^{1-4}$ An area of interest is the use of extracorporeal membrane oxygenation (ECMO) in patients with Fontan

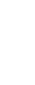

ongoing cardiogenic shock, an Impella CP (Abiomed Inc, Danvers, Mass) was placed, but she continued to have low systemic venous oxygen saturation in the 40 s in the setting of ongoing arrhythmias refractory to cardioversion and multiple antiarrhythmic medications despite normal common ventricle function. She was then cannulated for venoarterial (VA)-ECMO via an 18-Fr OptiSite cannula (Edwards Lifesciences, Irvine, Calif) in the right femoral artery with 6-Fr distal perfusion catheter and 23Fr Bio-Medicus cannula (Medtronic, Minneapolis, Minn) in the femoral vein, achieving flows of 3.5-4 liters per minute (patient weighed $110 \mathrm{lbs}$ ). The Impella was removed 3 days later. The patient was on VA-ECMO without intubation for 13 days before transplant. During this time, she was briefly deactivated due to spontaneous bacterial peritonitis; however, the infection was controlled, and she was able to be reactivated and received a suitable organ offer.

The heart-liver en bloc transplant was performed in similar fashion to that previously reported by our institution (Figure 1). ${ }^{5}$ For cardiopulmonary bypass, the existing right femoral ECMO cannula and an additional superior vena cava and portal vein cannula were used. Total bypass 

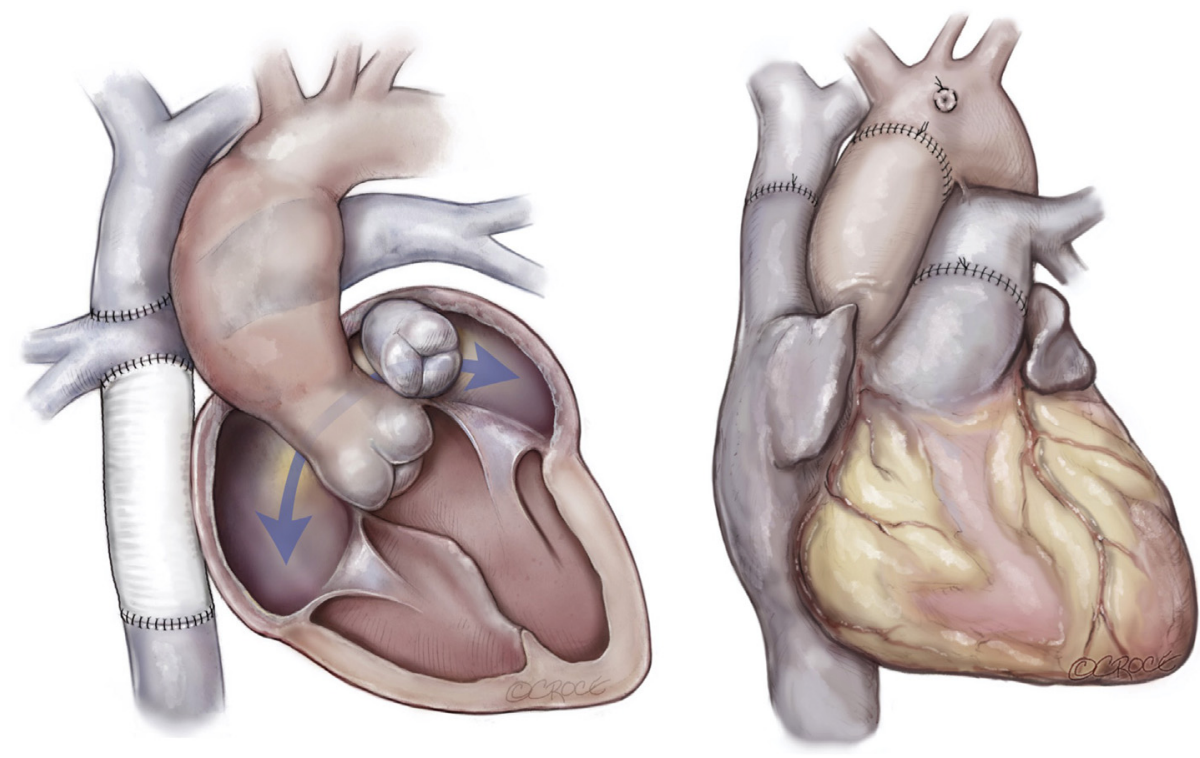

FIGURE 1. Pre- (left) and posttransplant (right) anatomy. Pretransplant, the patient had right ventricle dominant atrioventricular septal defect status post pulmonary artery banding as an infant followed by a combined Glenn procedure and extracardiac Fontan at age 22 years.

time was 195 minutes and donor organ ischemic time 231 minutes. She received protamine, $60+$ units of blood products, and an anti-inhibitor coagulant complex (FEIBA; Takeda Pharmaceutical Company Limited, Lexington, Mass) due to significant coagulopathy. This led to an elevated central venous pressure of 60 with right ventricular dysfunction when cardiopulmonary bypass was weaned. Therefore, the patient was placed back on VAECMO through the preoperative right femoral cannulas. The chest and abdomen were packed and left open. She returned for chest washout on postoperative day (POD) 1. Heparin drip was started 6 hours after the washout for ECMO anticoagulation.

The patient's chest and abdomen were closed on POD5, and she was stable for decannulation from VA-ECMO on POD6 (total of 19 days on VA-ECMO). She initially had moderate left ventricle dysfunction on VA-ECMO, but her donor heart was found to have normal function after decannulation. Although her postoperative course was complicated by gastrointestinal bleeding, tracheostomy on POD8, bilateral hemopneumothoraces, and renal failure (requiring continuous renal replacement therapy by POD5, now on intermittent hemodialysis at home and actively listed for kidney transplant), she has been discharged home and is now over 1 year out since her transplant.

\section{DISCUSSION}

We report here the first successful use of VA-ECMO in an adult patient with failing Fontan circulation as a bridge to heart-liver transplant. Although VA-ECMO has been used in patients with a failing Fontan as a bridge to ventricular assist device and heart transplant alone, it has not been used as a bridge to heart-liver en bloc transplant. The major concerns for ECMO in patients with Fontan circulation are achieving adequate venous drainage and ensuring afterload is not too high on the single ventricle when trying to achieve satisfactory ECMO flows. A straightforward cannulation strategy of femoral artery and vein allowed for adequate venous drainage and support of hemodynamics. ECMO afforded this patient additional time to overcome an acute insult on top of her failing Fontan physiology while preserving end organ perfusion, ultimately allowing her to remain a candidate for transplantation. As more patients with Fontan circulation reach adulthood and start to fail, institution of peripheral ECMO remains a viable option to stabilize and bridge these patients to transplant.

\section{CONCLUSIONS}

ECMO can be successfully used as a bridge to heart-liver en bloc transplantation in adult patients with failing Fontan circulation.

\section{References}

1. d'Udekem Y, Iyengar AJ, Galati JC, Forsdick V, Weintraub RG, Wheaton GR, et al. Redefining expectations of long-term survival after the Fontan procedure twenty-five years of follow-up from the entire population of Australia and New Zealand. Circulation. 2014;130:S32-8.

2. Book WM, Gerardin J, Saraf A, Valente AM, Rodriguez F III. Clinical phenotypes of Fontan failure: implications for management. Congenit Heart Dis. 2016;11: 296-308.

3. Poh CL, d'Udekem Y. Life after surviving Fontan surgery: a meta-analysis of the incidence and predictors of late death. Heart Lung Circ. 2018;27: $552-9$. 
4. Vaikunth SS, Concepcion W, Daugherty T, Fowler M, Lutchman G, Maeda K, et al. Short-term outcomes of en bloc combined heart and liver transplantation in the failing Fontan. Clin Transplant. 2019;33:e13540.
5. Nasirov T, Shudo Y, Concepcion W, Maeda K. En bloc combined heart and liver transplantation for end-stage heart failure. Oper Tech Thorac Cardiovasc Surg. 2021;26:112-7. https://doi.org/10.1053/j.optechstcvs.2020.07.003 
TABLE E1. Catheterization data from 2 months pretransplant and 1 month posttransplant

\begin{tabular}{lcc}
\hline Catheterization data & $\begin{array}{c}\text { 2 mo before } \\
\text { transplant }\end{array}$ & $\begin{array}{c}\text { 1-mo } \\
\text { posttransplant }\end{array}$ \\
\hline Inferior vena cava & $14 \mathrm{~mm} \mathrm{Hg}$ & N/A \\
Superior vena cava & $14 \mathrm{~mm} \mathrm{Hg}$ & N/A \\
Fontan & $13 \mathrm{~mm} \mathrm{Hg}$ & N/A \\
Right atrium & N/A & $6 / 9(5 \mathrm{~mm} \mathrm{Hg})$ \\
Right ventricle & N/A & $28 / 1(7 \mathrm{~mm} \mathrm{Hg})$ \\
Pulmonary artery & $13 \mathrm{~mm} \mathrm{Hg}$ (right side) & $24 / 9(16 \mathrm{~mm} \mathrm{Hg})$ \\
& $12 \mathrm{~mm} \mathrm{Hg}$ (left side) & \\
$\begin{array}{l}\text { Mean pulmonary } \\
\text { wedge }\end{array}$ & $9 \mathrm{~mm} \mathrm{Hg}$ (right side) & $9 \mathrm{~mm} \mathrm{Hg}$ \\
$\begin{array}{l}\text { Left ventricle } \\
\text { Aorta }\end{array}$ & $96 / 8 \mathrm{~mm} \mathrm{Hg}$ (left side) & N/A \\
$\begin{array}{l}\text { Fick cardiac index } \\
\text { The patient was not on any inotropes and did not have any mechanical circulatory sup- } \\
\text { port. } N / A, \text { Not available. }\end{array}$ & $96 / 52 \mathrm{~mm} \mathrm{Hg}$ & N/A \\
\hline
\end{tabular}

